\title{
Clustered Underwater Ad-Hoc Networks in the Presence of Interference
}

\author{
Andrej Stefanov and Milica Stojanovic \\ Electrical and Computer Engineering Department \\ Northeastern University \\ E-mail: a.stefanov@neu.edu, millitsa@mit.edu
}

\begin{abstract}
The paper considers the performance of clustered underwater acoustic ad-hoc networks in the presence of interference. We assume a uniform distribution of nodes over a finite area. The cluster-to-cluster channel is modeled as a Ricean fading channel. We adopt a communication theoretic approach and study the interdependence of the sustainable number of cluster-to-cluster hops through the network, end-to-end frame error probability, power and bandwidth allocation. We find that the network's ability to provide full connectivity may be limited from below by coverage and from above by interference. The network may be coverage-limited when the number of nodes in the network is small, and interference-limited when the number of nodes is high. Numerical examples are presented that illustrate the results of the analysis.
\end{abstract}

\section{INTRODUCTION}

Recently, there has been tremendous progress in the analysis and the design of underwater wireless (acoustic) communications systems [1]. The development of underwater monitoring systems, however, also requires an understanding of underwater networking principles. Therefore there is a need for a study and an analysis of underwater acoustic networks.

The performance of underwater acoustic ad-hoc networks was addressed in [2] and [3]. Specifically, the study in [3] was performed under the assumption that the nodes in the network are organized into clusters forming virtual transmit/receive arrays [4]. This represents an appealing alternative to standard multihop transmission [2], since by utilizing suitable signal processing techniques the virtual transmit/receive arrays have the potential to deliver the power savings and rate/reliability benefits of multiple-input multiple-output (MIMO) systems. Both these studies, however, were conducted under the idealized assumption that there is no interference in the network.

In this paper, we extend the results of [3] to include the effect of interference. We focus on an underwater network of bottom mounted nodes, thus we consider a two-dimensional network model. We assume multihop transmission based on nearest neighbor cluster-to-cluster routing, as it offers more beneficial bandwidth and path loss conditions. We adopt a communication theoretic approach [5] and investigate the network performance in the presence of interference from other clusters in the network. We focus on the interdependence between the sustainable number of cluster-to-cluster hops in the network as an indicator of network connectivity, end-toend frame error probability, power and bandwidth allocation.
The paper is organized as follows. Section II describes the underwater acoustic propagation model. Section III describes the clustered ad-hoc network set-up and the communication theoretic analysis. Numerical results are presented in section IV. We conclude with section V.

\section{Underwater ACOUstic Propagation}

\section{A. Attenuation}

Attenuation, or path loss, that occurs in an underwater acoustic channel over a distance $d$ for a signal of frequency $f$, is given by

$$
A(d, f)=A_{0} d^{\kappa} a(f)^{d}
$$

where $A_{0}$ is a unit-normalizing constant, $a(f)$ is the absorption coefficient and $\kappa$ is the spreading factor [6]. In the case of practical spreading $\kappa=1.5$. The absorption coefficient can be expressed empirically, using Thorp's formula which gives $a(f)$ in $\mathrm{dB} / \mathrm{km}$ for $f$ in $\mathrm{kHz}$ as

$$
10 \log a(f)=\frac{0.11 f^{2}}{1+f^{2}}+\frac{44 f^{2}}{4100+f^{2}}+\frac{2.75 f^{2}}{10^{4}}+0.003 .
$$

This formula is generally valid for frequencies above a few hundred $\mathrm{Hz}$.

\section{B. Noise}

The ambient noise in the ocean can be modeled using four sources: turbulence, shipping, waves and thermal noise, which can be described by Gaussian statistics and a continuous power spectral density. The following empirical formulae give the power spectral densities of the noise components in $\mathrm{dB}$ re $\mu \mathrm{Pa}$ per $\mathrm{Hz}^{1}$ as a function of frequency in $\mathrm{kHz}$ [6]

$$
\begin{aligned}
10 \log N_{\mathrm{t}}(f)= & 17-30 \log f, \\
10 \log N_{\mathrm{s}}(f)= & 40+20(s-0.5)+26 \log f \\
& -60 \log (f+0.03), \\
10 \log N_{\mathrm{w}}(f)= & 50+7.5 \sqrt{w}+20 \log f \\
& -40 \log (f+0.4), \\
10 \log N_{\mathrm{th}}(f)= & -15+20 \log f
\end{aligned}
$$

where $s$ is the shipping activity factor, $0 \leq s \leq 1$, and $w$ is the wind speed in $\mathrm{m} / \mathrm{s}$. The overall power spectral density of the ambient noise is $N(f)=N_{\mathrm{t}}(f)+N_{\mathrm{s}}(f)+N_{\mathrm{w}}(f)+N_{\mathrm{th}}(f)$.

\footnotetext{
${ }^{1}$ Note that an acoustic signal propagates as a pressure wave whose level is commonly measured in $\mathrm{dB}$ relative to $1 \mu \mathrm{Pa}$.
} 


\section{Ad-hoc Network Setup}

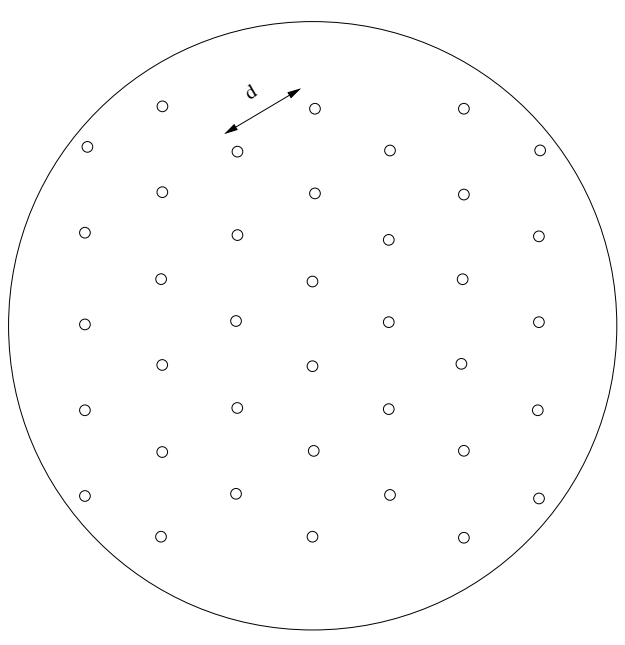

Fig. 1. Uniform network coverage.

\section{A. Network Topology}

We consider a network of bottom mounted nodes. Therefore, we focus on a two dimensional network that provides coverage over a certain area. We assume that the area of the network is a circle and consider a uniform distribution of nodes in the network as depicted in Figure 1. Given the total number of nodes in the network, $N$, and the area of the network, $\mathcal{A}$, the density of the network is $\rho_{s}=\frac{N}{\mathcal{A}}$. The distance between nodes is $d=\frac{c}{\sqrt{\rho_{s}}}$, where $c$ is a constant that depends on the node placement (grid pattern). Without loss of generality we assume that $c=1$.

We assume that groups of $N_{c}$ nodes are organized into clusters. We define the distance between clusters as the distance between the centers of the clusters

$$
d_{c} \approx d \sqrt{N_{c}} \approx \sqrt{\frac{N_{c}}{\rho_{s}}} .
$$

We assume cluster-to-cluster multihop routes along nearest neighbor clusters, as depicted in Figure 2. Clustering is an energy saving strategy, hence it may be attractive for networks with battery powered nodes. As the longest multihop route in the network is along the diameter of the network, $D=2 \sqrt{\mathcal{A} / \pi}$, the maximum number of cluster-to-cluster hops is

$$
n_{h}^{\max }=\frac{D}{d_{c}}=\frac{2}{\sqrt{\pi}} \sqrt{\frac{N}{N_{c}}} .
$$

Let the average number of cluster-to-cluster hops for a multihop route be denoted as $\bar{n}_{h}$. Then, as long as the probability distribution for the number of hops is symmetric, that is, as long as very long and very short routes are much less likely than routes with an average number of cluster-to-cluster hops, we have [5]

$$
\bar{n}_{h}=\frac{n_{h}^{\max }}{2}=\frac{1}{\sqrt{\pi}} \sqrt{\frac{N}{N_{c}}} .
$$

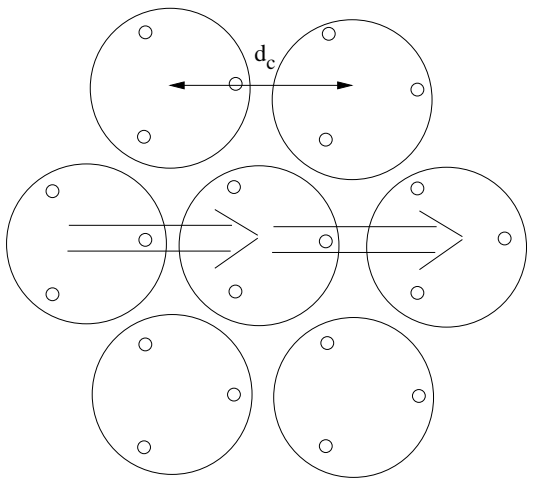

Fig. 2. Cluster-to-cluster transmission with $N_{c}=3$ nodes per cluster.

\section{B. Interference Model}

In order to illustrate the interference model, we focus on a single transmission from a source cluster to a destination cluster, as depicted in Figure 3. We impose a protocol constraint: no clusters that are at the same distance from the destination cluster as the source cluster are allowed to transmit in the same time slot and in the same frequency band as the source during the source cluster's transmission.

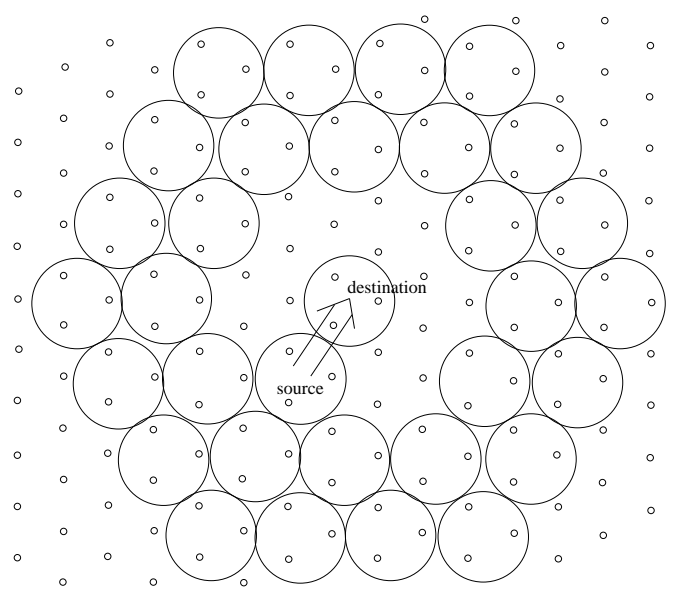

Fig. 3. Interfering clusters in the network.

The remaining clusters that may interfere with the source cluster's transmission are organized in tiers. As we assume hexagonal topology, there will be at most 12 interfering clusters in tier 1 , and 18 interfering clusters in tier 2 , as illustrated in Figure 3. A scenario where all the other clusters in the network transmit at the same time would be unrealistic, as we also need to consider that some clusters will be receiving. Assuming that all clusters transmit at some constant power spectral density (p.s.d.) $S$, the interference from the clusters in the first and in the second tier is

$$
I(f) \approx \frac{c_{1} S}{A\left(2 d_{c}, f\right)}+\frac{c_{2} S}{A\left(3 d_{c}, f\right)}
$$

where $c_{1} \leq 12$ and $c_{2} \leq 18$ are constants indicating the number of interfering clusters in tier 1 and tier 2, respectively. In 
particular, we let $c_{1}=c_{2}=6$. As there are multiple interfering clusters in the network, we assume that the interference is Gaussian with a p.s.d. given by Eq. (7).

Using the attenuation $A(d, f)$, the noise p.s.d. $N(f)$ and the interference p.s.d. $I(f)$, we can evaluate the signal to interference plus noise ratio (SINR) observed over a distance $d_{c}$. The SINR is shown in Figure 4. We observe that there is a preferred operating frequency, $f_{o}\left(d_{c}\right)$, which depends on the distance, $d_{c}$, where $\left[A\left(d_{c}, f\right)(N(f)+I(f))\right]^{-1}$ is maximized. Figure 5 presents this preferred operating frequency, $f_{o}\left(d_{c}\right)$, as a function of the distance, given a transmit p.s.d. level of $S=110 \mathrm{~dB}$ re $\mu \mathrm{Pa}$ per $\mathrm{Hz}$ for $f$ in $\mathrm{kHz}$. We note that the preferred operating frequency is higher when there is interference.

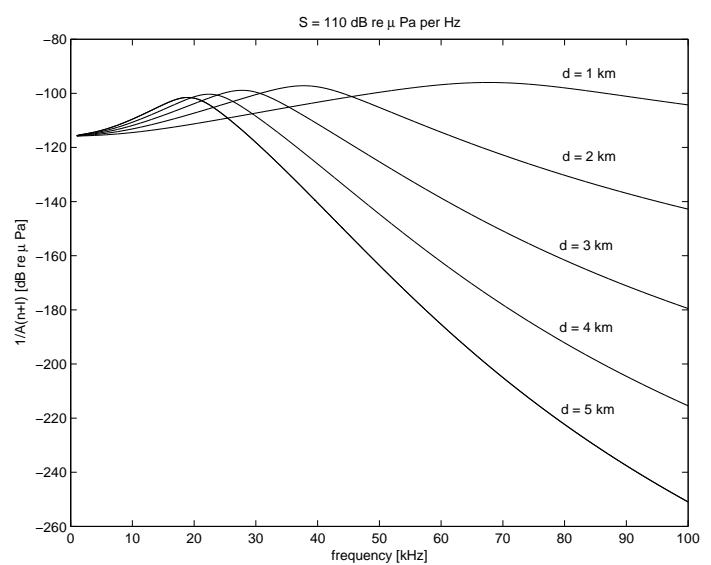

Fig. 4. The signal to noise plus interference ratio for various distances $d$. The transmit power spectral density is $S=110 \mathrm{~dB}$ re $\mu \mathrm{Pa}$ per Hz. The spreading factor is $\kappa=1.5$.

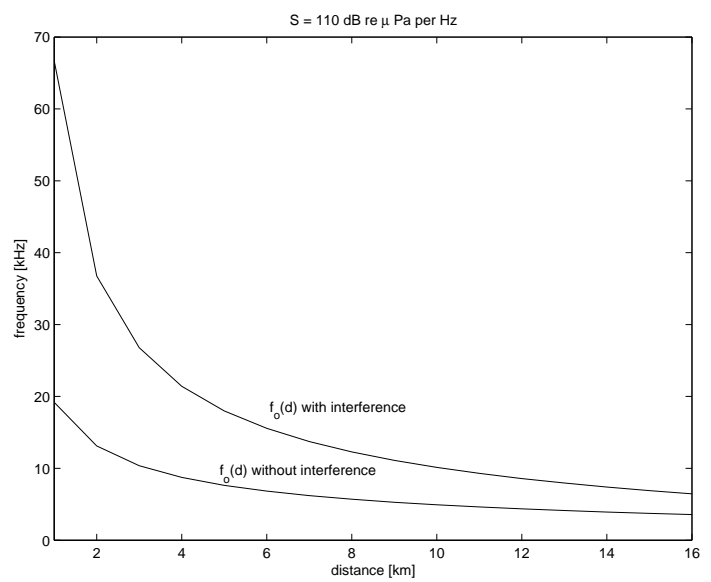

Fig. 5. Operating frequency $f_{o}\left(d_{c}\right)$. The transmit power spectral density is $S=110 \mathrm{~dB}$ re $\mu \mathrm{Pa}$ per Hz. The spreading factor is $\kappa=1.5$.

We assume a simple distributed space-time block code with a decode and forward relaying strategy. The end-to-end frame error probability (FEP) for a multihop route with $n_{h}$ clusterto-cluster hops, $p_{\text {route }}$, is given by

$$
p_{\text {route }}=1-\left(1-p_{b}\right)^{L n_{h}}
$$

where $p_{b}$ denotes the bit error probability of a single clusterto-cluster link and $L$ denotes the frame size in bits.

We consider the quality-of-service for the network in terms of the maximum allowed end-to-end route FEP, i.e., we require that $p_{\text {route }} \leq p_{\text {route }}^{\max }$. Let the number of cluster-to-cluster hops that can be sustained by the network, i.e., the number of cluster-to-cluster hops that can satisfy the maximum end-toend route FEP, be denoted by $n_{\text {sh }}$. From Eq. (8), it follows that $n_{\text {sh }}$ can be calculated as ${ }^{2}$

$$
n_{\text {sh }}=\frac{1}{L} \frac{\log \left(1-p_{\text {route }}^{\max }\right)}{\log \left(1-p_{b}\right)} \approx \frac{1}{L} \frac{p_{\text {route }}^{\max }}{p_{b}} .
$$

Under the assumption of a Ricean fading model for the cluster-to-cluster channel [7], and assuming that perfect channel state information is available at the receiver, the bit error probability can be approximated as [8]

$$
p_{b} \lesssim\left(\frac{1+\mathcal{K}}{1+\mathcal{K}+\gamma\left(d_{c}, f\right)}\right)^{t r} \exp \left(-\frac{\operatorname{tr} \mathcal{K} \gamma\left(d_{c}, f\right)}{1+\mathcal{K}+\gamma\left(d_{c}, f\right)}\right)
$$

where $\mathcal{K}$ denotes the Ricean fading factor assumed to be the same for all node-to-node sub-channels, $t$ denotes the transmit diversity gain, $r$ denotes the receive diversity gain and $\gamma$ denotes the SINR. Note that $1 \leq t, r \leq N_{c}$. We assume that the attenuation, noise and interference are constant over the entire bandwidth, so that the SINR can be calculated as

$$
\gamma\left(d_{c}, f\right)=\frac{S}{A\left(d_{c}, f\right)(N(f)+I(f))} .
$$

The frequency-nonselective assumption is a suitable approximation for systems with narrow bandwidth. It can also be extended to wideband multi-carrier systems, such as OFDM [9]. In that case, the operating frequency, $f_{o}\left(d_{c}\right)$, would describe the performance on one of the carriers. The performance on the other carriers would correspond to the frequency $f_{o}\left(d_{c}\right)$ shifted by multiples of subcarrier separation $\Delta f$.

\section{NUMERICAL RESULTS}

We present numerical examples that examine the relationships between the sustainable number of cluster-to-cluster hops, end-to-end FEP, signal power and bandwidth. We assume Ricean fading for each cluster-to-cluster channel with $\mathcal{K}=10$ for all node-to-node sub-channels. We assume a circular network of area $\mathcal{A}=1000 \mathrm{~km}^{2}$. We assume the attenuation model given in Eq. (1) and neglect any fixed losses. Inclusion of additional frequency-independent losses, and adjustment of the background noise level to suit a particular environement and provide the necessary SINR margins, will scale the results in absolute value, but will not alter the general behavior. The frame size is $L=1000$ bits. The spreading factor is $\kappa=1.5$, the shipping activity factor is $s=0.5$, and the wind speed is $w=0 \mathrm{~m} / \mathrm{s}$.

Figure 6 presents the sustainable number of clusterto-cluster hops for a maximum allowed end-to-end FEP

\footnotetext{
${ }^{2}$ Note that while the analysis does not consider it explicitly, in practice $\left\{n_{\mathrm{sh}}, n_{h}^{\max }\right\} \in \mathbb{N}$.
} 
of $10^{-3}$, the bandwidth $B=4 \mathrm{kHz}$ and the transmit power $P=108 \mathrm{~dB}$ re $\mu \mathrm{Pa}$, where $P=S B$. The cluster size is $N_{c}=3$. We assume that $t=3$ and $r=1$, that is, the nodes in the transmit cluster collaborate to form a distributed spacetime code, but a single node acts as a receiver in the receiving cluster. The nodes are able to adjust their powers, so that the sustainable number of hops through the network never exceeds the maximum number of cluster-to-cluster hops given in Eq. (5). The average number of cluster-to-cluster hops given by Eq. (6) is also presented. We observe that when there are fewer than $N \lesssim 1600$ nodes, the network cannot provide full connectivity. This is due to the fact that with so few nodes in the network, the nodes are too far apart to guarantee the required end-to-end FEP for the available transmit power. Hence, the network is coverage-limited. Contrary to this situation, when the number of nodes in the network is above $N \gtrsim 7500$, we observe that the network can no longer sustain routes with the maximum number of hops. As we increase the number of nodes, while keeping the area of the network constant, the distance between the nodes decreases and the interference becomes stronger. Hence, the sustainable number of hops begins to decrease. We note, however, that even with $N=10000$ nodes the network can still maintain routes with an average number of hops. Nonetheless, the network is interference-limited. When the number of nodes in the network is between these values, $1600 \lesssim N \lesssim 7500$, the network can provide full connectivity and meet the target end-to-end FEP.

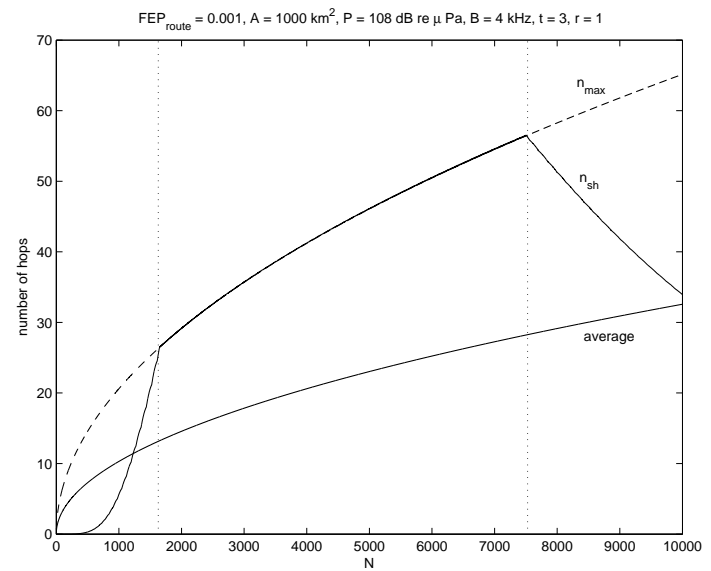

Fig. 6. Sustainable number of cluster-to-cluster hops for a uniform network with Ricean fading. The area is $\mathcal{A}=1000 \mathrm{~km}^{2}$, the bandwidth is $B=4$ $\mathrm{kHz}$, the transmit power is $P=108 \mathrm{~dB}$ re $\mu \mathrm{Pa}$, the cluster size is $N_{c}=3$, $t=3, r=1$.

Figure 7 presents the sustainable number of cluster-tocluster hops for different values of the transmit power. The required end-to-end FEP is $10^{-3}$. The cluster size is $N_{c}=3$, $t=3, r=1$. The bandwidth is $B=4 \mathrm{kHz}$. When the power is $P=120 \mathrm{~dB}$ re $\mu \mathrm{Pa}$ the network provides full connectivity for all values of $N<10000$. If the power is decreased to $P=108 \mathrm{~dB}$ re $\mu \mathrm{Pa}$, the network provides full connectivity when the number of nodes in the network is between $1600 \lesssim N \lesssim 7500$. When the power is further decreased to $P=105 \mathrm{~dB}$ re $\mu \mathrm{Pa}$, we observe that the network cannot support the average number of cluster-to-cluster hops for any number of nodes.

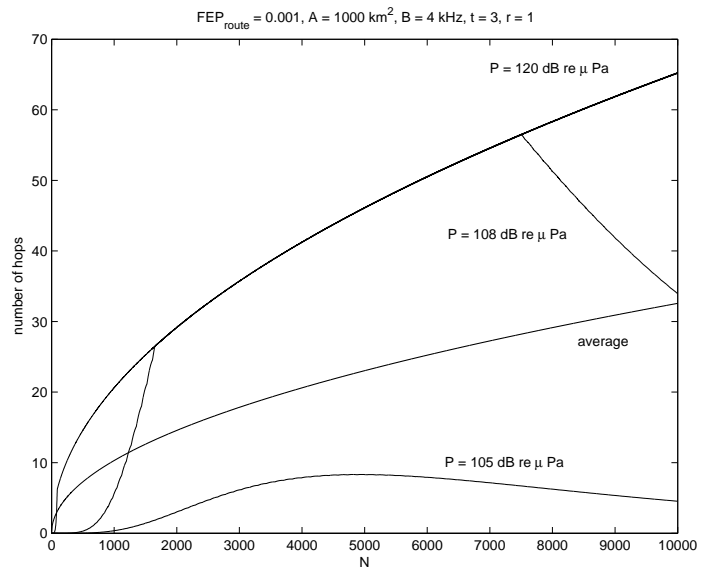

Fig. 7. Sustainable number of cluster-to-cluster hops for a uniform network with Ricean fading for various powers. The area is $\mathcal{A}=1000 \mathrm{~km}^{2}$, the bandwidth is $B=4 \mathrm{kHz}$, the cluster size is $N_{c}=3, t=3, r=1$.

Figure 8 depicts the sustainable number of cluster-to-cluster hops for different values of the bandwidth. The end-to-end FEP is $10^{-3}$. The power is $P=108 \mathrm{~dB}$ re $\mu \mathrm{Pa}$. The cluster size is $N_{c}=3, t=3, r=1$. We observe that the network behavior changes as we vary the bandwidth. When the bandwidth is $B=1 \mathrm{kHz}$ the network provides full connectivity when the number of nodes is between $250 \lesssim N<10000$. When the bandwidth is increased to $B=4 \mathrm{kHz}$, the network becomes interference-limited when the number of nodes is $N \gtrsim 7500$ and coverage-limited when the number of nodes is $N \lesssim 1600$. Note that this behavior is not inherent to the channel, but is rather a consequence of the fact that we have used the same signal power in both cases. In other words, while the signal power remains the same, the noise power increases with the increased bandwidth causing an overall degradation in the system performance.

The sensitivity of the sustainable number of cluster-tocluster hops to the carrier frequency is addressed in Figure 9. The end-to-end FEP is $10^{-3}$, the bandwidth is $B=4 \mathrm{kHz}$ and the transmit power is $P=108 \mathrm{~dB}$ re $\mu \mathrm{Pa}$. The cluster size is $N_{c}=3, t=3, r=1$. We observe that when $f_{o}$ is chosen as the operating frequency, the network becomes coverage-limited when the number of nodes is $N \lesssim 1600$, and interference-limited when the number of nodes is $N \gtrsim 7500$. If the carrier frequency is $f_{o} \pm 5 \mathrm{kHz}$, we observe that the network becomes coverage-limited when the number of nodes in the network is $N \lesssim 3600$, and interference-limited when the number of nodes in the network is $N \gtrsim 6300$. The network behaves in this way because a deviation from the preferred operating frequency $f_{o}$ causes the SINR to decrease, as depicted in Figure 4, and consequently, the sustainable number of hops decreases as well. Hence, in order to ensure the widest range of supported node densities, it is necessary to choose the operating frequency that maximizes the SINR. 


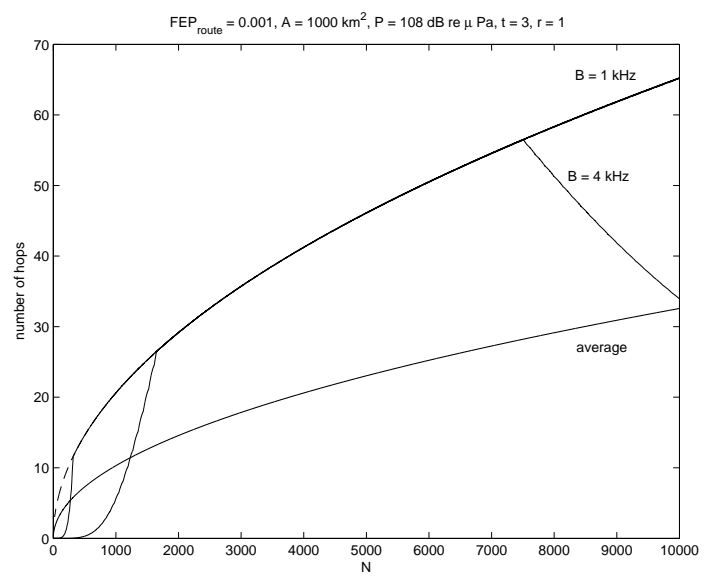

Fig. 8. Sustainable number of cluster-to-cluster hops for a uniform network with Ricean fading for various bandwidths. The area is $\mathcal{A}=1000 \mathrm{~km}^{2}$, the transmit power is $P=108 \mathrm{~dB}$ re $\mu \mathrm{Pa}$, the cluster size is $N_{c}=3, t=3$, $r=1$.

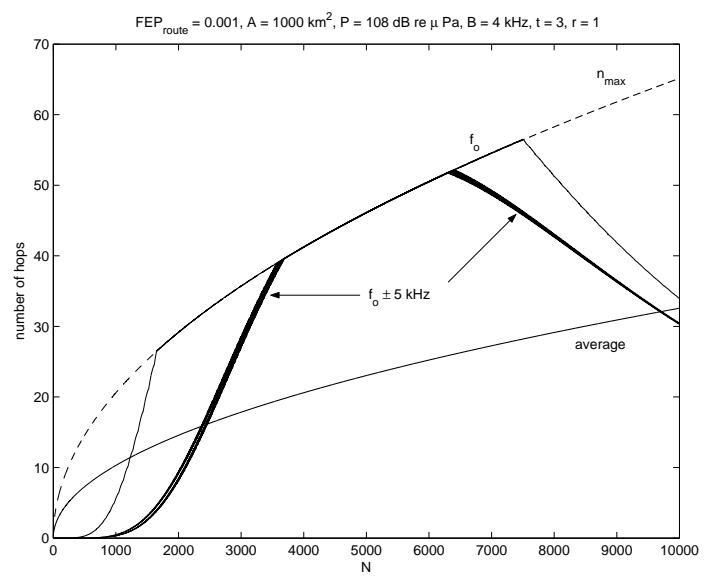

Fig. 9. Sustainable number of cluster-to-cluster hops for a uniform network with Ricean fading for various carrier frequencies: $f_{o}$ and $f_{o} \pm 5 \mathrm{kHz}$. The area is $\mathcal{A}=1000 \mathrm{~km}^{2}$, the bandwidth is $B=4 \mathrm{kHz}$, the transmit power is $P=108 \mathrm{~dB}$ re $\mu \mathrm{Pa}$, the cluster size is $N_{c}=3, t=3, r=1$.

Figure 10 presents the sustainable number of cluster-tocluster hops for various diversity levels. The end-to-end FEP is $10^{-3}$, the bandwidth is $B=4 \mathrm{kHz}$, the transmit power is $P=108 \mathrm{~dB}$ re $\mu \mathrm{Pa}$, and the cluster size is $N_{c}=3$. When the diversity level is 3 , i.e., $t=3$ and $r=1$, the network is coverage-limited for $N \lesssim 1600$, and interference-limited for $N \gtrsim 7500$. When the diversity level is increased to 4, i.e., $t=r=2$, the network is no longer interference-limited. It is still coverage-limited when the number of nodes is $N \lesssim 500$. Finally, when the virtual arrays achieve full transmit and receive diversity, i.e., $t=r=3$, the network is neither coverage-limited, nor interference-limited.

\section{Conclusions}

We considered a two dimensional network of bottom mounted nodes. The nodes were organized as clusters forming virtual transmit/receive arrays. The cluster-to-cluster channel was modeled as a Ricean fading channel. A communication

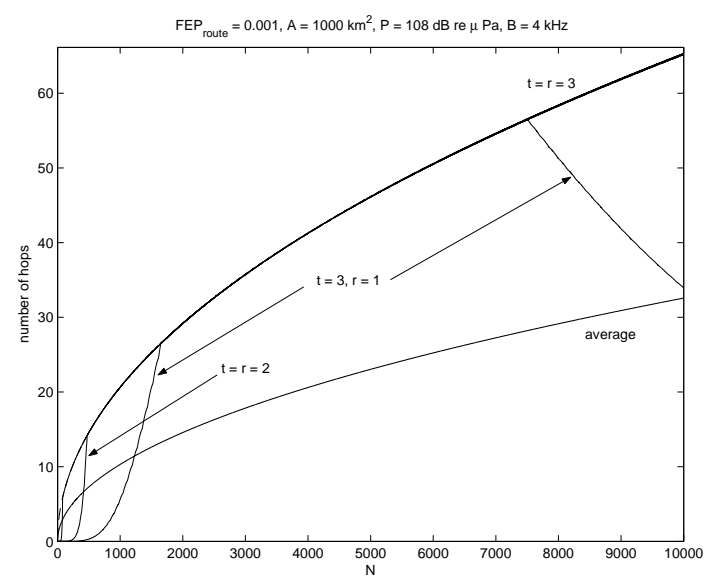

Fig. 10. Sustainable number of cluster-to-cluster hops for a uniform network with Ricean fading for various diversity levels. The area is $\mathcal{A}=1000 \mathrm{~km}^{2}$, the transmit power is $P=108 \mathrm{~dB}$ re $\mu \mathrm{Pa}$, the bandwidth is $B=4 \mathrm{kHz}$, the cluster size is $N_{c}=3$.

theoretic analysis of the network was conducted in the presence of interference to assess the sustainable number of hops in varying conditions. The sustainable number of hops in turn provided an indication of the number of nodes that can be supported in a given area, with given resources (power), and a desired quality of performance (FEP). When the number of nodes in the network is too small, such that the available power is not sufficient to provide connectivity over a (large) area, the network is coverage-limited. As the number of nodes in the network increases while the network area remains constant, the distance between the nodes decreases, and the network becomes interference-limited. Both the coverage-limited region and the interference-limited region can be controled through a proper choice of operating frequency, transmit power, and diversity level achieved by the virtual transmit/receive array.

\section{ACKNOWLEDGMENTS}

This work was supported in part by the NSF grant 0831728 and the ONR grant N00014-09-1-0700.

\section{REFERENCES}

[1] IEEE Journal on Selected Areas in Communications, Special Issue on Underwater Wireless Communications and Networks, December 2008.

[2] A. Stefanov and M. Stojanovic, "Performance of Underwater Ad-Hoc Networks," in Proc. IEEE VTC-Fall, September, 2010.

[3] A. Stefanov and M. Stojanovic, "Clustered Multihop Transmission in Underwater Acoustic Ad-Hoc Networks," in Proc. IEEE ICCS, November, 2010.

[4] M. Dohler, "Virtual Antenna Arrays," Ph.D. Thesis, King's College, London, UK, 2003.

[5] O. Tonguz and G. Ferrari, "Ad Hoc Wireless Networks: A Communication-Theoretic Perspective," Wiley, 2006.

[6] L. Berkhovskikh and Y. Lysanov, "Fundamentals of Ocean Acoustics," Springer, 1982.

[7] P. Qarabaqi and M. Stojanovic, "Statistical Modeling of a Shallow Water Acoustic Communication Channel," in Proc. Underwater Acoustic Measurements Conference, Nafplion, Greece, June, 2009.

[8] S. Benedetto and E. Biglieri, "Principles of Digital Transmission with Wireless Applications," Kluwer/Plenum, 1999.

[9] L. Hanzo and T. Keller, "OFDM and MC-CDMA: A Primer," WileyIEEE Press, 2006. 